\title{
Is the Inhibition/Enhancement of Fusion Due to Breakup Still a Puzzle?
}

\author{
A. Szanto de Toledo, M.G. Munhoz, J. Takahashi, F.A. Souza, E.E. Alonso, \\ N. Carlin, R. Liguori Neto, M.M. Moura, A.A.Suaide, and E.M. Szanto \\ Departamento de Física Nuclear, Laboratório Pelletron, \\ Universidade de São Paulo, C.P. 66318, 5315-970 São Paulo, Brazil
}

Received on 30 October, 2002

\begin{abstract}
The effect of breakup in the fusion cross section in terms of suppression versus enhancement, discussed in a conflicting way in the literature, is addressed. Data and theoretical predictions available in the literature are compared. Excitation functions of the sub- and near-barrier fusion cross-sections for a wide variety of light and heavy systems are presented and interpreted. We have measured fusion excitation functions and breakup correlation functions for the medium weight systems ${ }^{6} \mathrm{Li}+{ }^{59} \mathrm{Co}$ and ${ }^{7} \mathrm{Li}+{ }^{59} \mathrm{Co}$. These measurements help to establish the influence of the projectile breakup on the fusion process at near-barrier energies and contribute to the determination of how the mass of the target affects the breakup role. The results indicate a light fusion enhancement at sub-barrier energies and a geometry dominated cross section at barrier energies.
\end{abstract}

Systematic experimental and theoretical studies on the effect of the coupling of collective degrees of freedom to the fusion process, which lead to a significant enhancement of sub-barrier fusion cross-section when compared to predictions of one dimensional barrier penetration models, have been, so far, widely reported in the literature (ref 1-3). The dynamical process leading to this enhancement seems to be well understood (3). However, in the case of reactions where at least one of the colliding nuclei has a very low binding energy, and the breakup process may become an important reaction channel, conflicting model predictions and experimental results are found (4-7).

Many questions regarding this feature became more relevant due to the large impact they may have on the investigations made possible with the recent availability of radioactive beams and the renewed interest in superheavy elements. It is so far established that the coupling of collective additional degrees of freedom to the sub-barrier fusion channel systematically enhances the Complete Fusion (CF) cross section. However, the theoretically expected decrease of the survival probability of weakly bound nuclei at barrier energies suggests an inhibition of the CF cross section. Experimental results have shown that the nuclear breakup channel may be a major limiting process for the fusion probability above the barrier energy when light weakly bound nuclei $(8,9)$ are involved. On the other hand, the Coulomb breakup may also be important when weakly bound nuclei interact with very massive heavy-ions systems (10-12). One open and interesting question that remains in the case involving medium weight nuclei is whether the breakup process hinders or enhances fusion cross sections in different energy regimes.
Recently a systematic study of the fusion process in systems as light as ${ }^{6,7} \mathrm{Li}+{ }^{12} \mathrm{C}$ and ${ }^{6,7} \mathrm{Li}+{ }^{9} \mathrm{Be}$ has been $(8,13,14)$ presented reporting, at energies $\mathrm{E}$ above the barrier $V_{B}$, $\left(E>2 V_{B}\right)$, a correlation between the participants binding energy, the size of the system and the degree of inhibition of the fusion probability. This study indicated that the degree of inhibition of the fusion probability results from two adding phenomena. One directly related to the lowest binding energy for particle or cluster breakup, and another related to the size of the system by the ratio of skin / core volume. (14). Conflicting interpretations reported in the litterature, are, in part, originated from the use of different references to state that an inhibition is observed: reaction cross section, predictions based on one dimentional barrier penetration model (1BPM), fusion cross section of similar systems, and others.

From the theoretical point of view, this problem also rises interesting and intriguing questions. Some approaches predict an enhancement of the fusion yield, whereas others predict the suppression of fusion. Furtermore, other models predict the coexistence of both features as sub-barrier enhancement and suppresion above the barrier.

Very recently, Tripathi et al (12) indicated that the competition between the enhancement and inhibition factors are resultant of coupling into the fusion channel, rather than an incoherent survival probability of the entrance channel.

The studies presented in the literature are concentrated in two different domains: light systems dominated by single particle and geometrical effects, and massive systems dominated by Coulomb interactions and collective behaviour.

The effect on medium weight systems, which represent an intermediate configuration and for which the geometrical 
attenuation of fusion and Coulomb breakup processes may compete equally, has not been addressed yet in the litterature. Within this scenario we have extended the study of the effect of the breakup on fusion cross sections by measuring sub- and near-barrier fusion cross sections for the ${ }^{6} \mathrm{Li}+{ }^{59} \mathrm{Co}$ and ${ }^{7} \mathrm{Li}+{ }^{59} \mathrm{Co}$ reactions. This measurement involves a significantly heavier target than the previous work of Takahashi et al (8), and lighter than the one used by Dasgupta et al. (11), thus enabling to explore to what extend the influence of the breakup channel scales with the target mass. This is an important consideration in planning subsequent near barrier fusion experiments with radioactive beams.

The ${ }^{6,7} \mathrm{Li}$ beams, of energies between 11 and $26 \mathrm{MeV}$ and of intensity of 5-10 pnA, were provided by both the Uni- versity of São Paulo 8UD Pelletron Tandem facility and the VIVITRON electrostatic Tandem accelerator of the Institut de Recherches Subatomiques (IReS), Strasbourg. The fusion cross sections were obtained through the detection of the gamma rays related to the evaporation residues.

We have also searched for Incomple Fusion ( ICF) yields at the highest energy data. Hauser-Feshbach calculations using the statistical-model code CASCADE (18) were used to identify and /or check possible contamination of specific lines associated to evaporation residues with contributions from Incomplete Fusion process. The critical angular momenta have been deduced from the experimental total fusion cross sections using the sharp cut-off approximation.
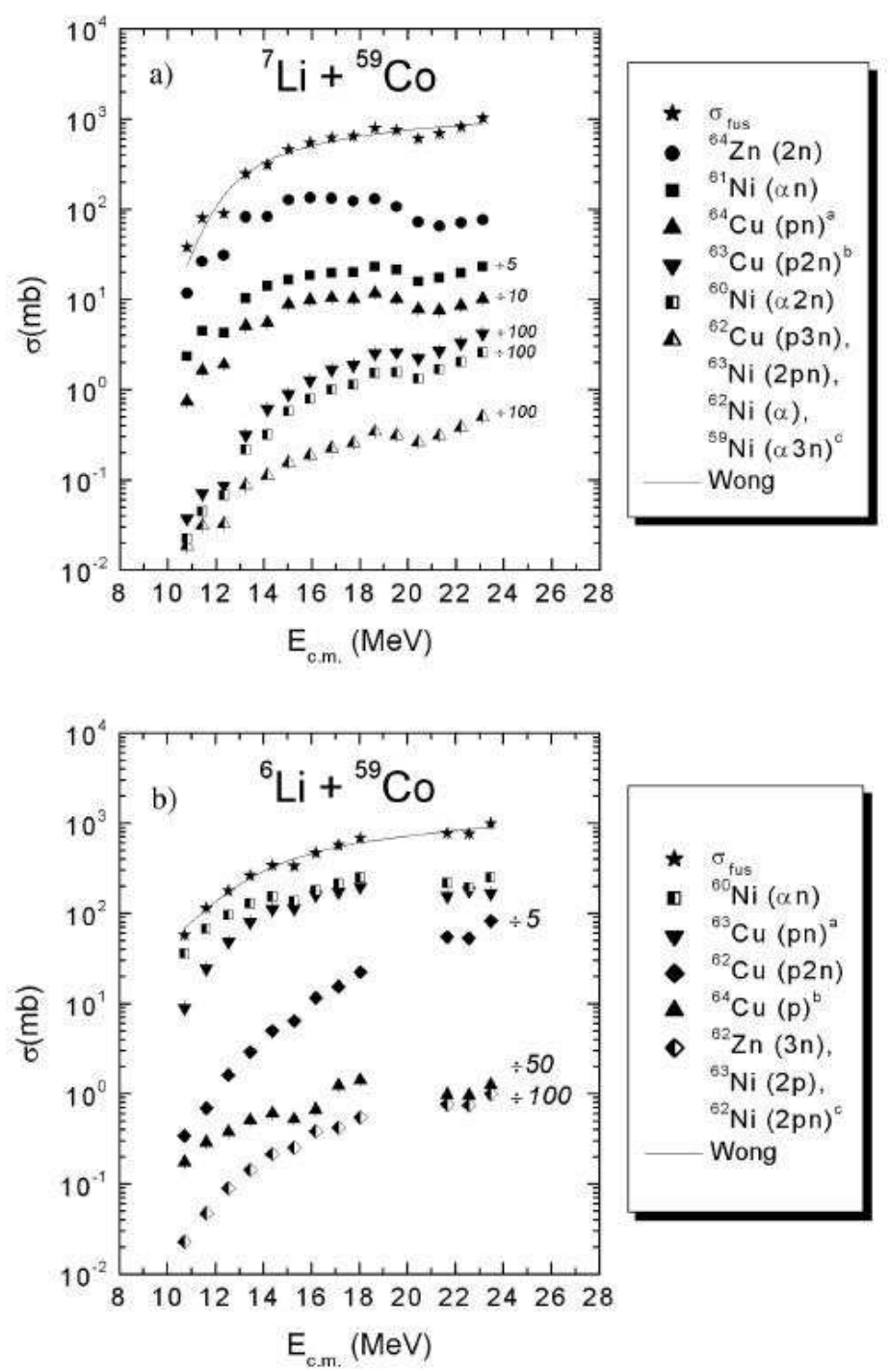

Figure 1. a) Excitation functions for the most proeminent fusion-evaporation exit-channels of the ${ }^{7} \mathrm{Li}+{ }^{59} \mathrm{Co}$ reaction. The upper excitation function represents the sum of all the observed decay channels, associated to the total fusion cross section. b) same for the ${ }^{6} \mathrm{Li}+{ }^{59} \mathrm{Co}$ reaction. 
The average excitation functions for the fusion cross section (Fig. 1), were fitted to 1BPM. In order to identify possible deviations from the expected behaviour due to the coupling of specific processes ( breakup in this case) and minimizing the geometrical effects, the fusion cross section of the ${ }^{7} \mathrm{Li}+{ }^{59} \mathrm{Co}$ channel $\left(\sigma_{F}\left({ }^{7} \mathrm{Li}\right)\right)$ is used as reference. Consequently, the ratio of the total fusion cross sections $\sigma_{F}$ between both reactions $\left\{\mathrm{R}=\sigma_{F}\left({ }^{6} \mathrm{Li}\right) / \sigma_{F}\left({ }^{7} \mathrm{Li}\right)\right\}$ as a function of the energy $\mathrm{E}_{\text {c.m. }}$., determines the intensity of the dynamical effects (see Fig. 2). Due to the fact that both systems present very similar entrance channel asymmetries, this ratio removes the geometrical dependence of the cross section, enhancing the effect of coupling to specific reaction channels on the fusion process $(4,7,12)$. A slight enhancement of the fusion process is apparent below the Coulomb barrier. On the other hand at energies above the barrier no suppression is observed for the ${ }^{6} \mathrm{Li}+{ }^{59} \mathrm{Co}$ system within the experimental uncertainties $(6,7,8)$.

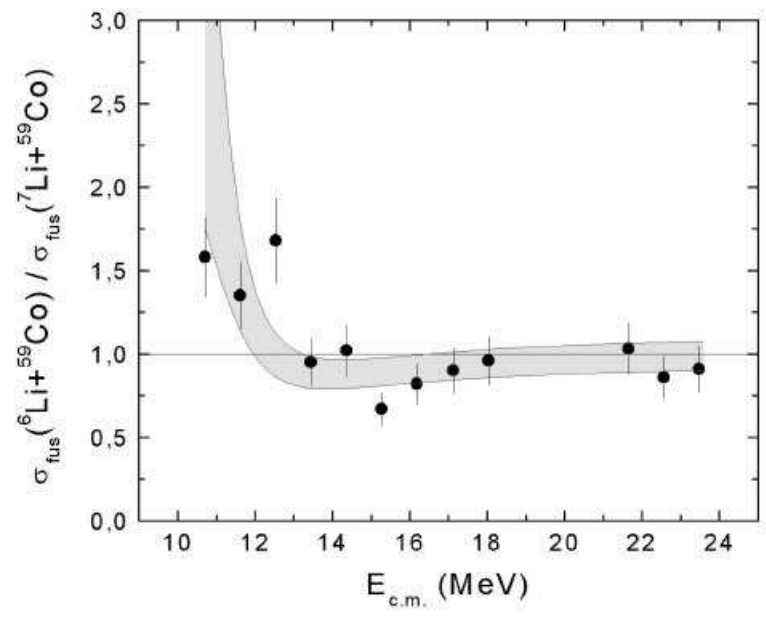

Figure 2. Energy dependence of ratio $\mathrm{R}$ of between the total fusion cross sections of ${ }^{6} \mathrm{Li}+{ }^{59} \mathrm{Co}$ and ${ }^{7} \mathrm{Li}+{ }^{59} \mathrm{Co}\left(R=\sigma_{F}\left({ }^{6} \mathrm{Li}\right) / \sigma_{F}\left({ }^{7} \mathrm{Li}\right)\right)$ The width of the band containing the experimental points represents the uncertainty deduced from the statistical and systematic uncertainties of the data.

This lack of significant suppression, was expected for this system as predicted by the systematic study presented in reference 14. In this figure it becomes clear that significant suppression is expected for weakly bound nuclei $(\varepsilon<2$ $\mathrm{MeV})$ and systems of size $A_{1}^{1 / 3}+A_{2}^{1 / 3}<4.8$ equivalent to ${ }^{7} \mathrm{Li}+{ }^{24} \mathrm{Mg}$.

Systems heavier than this one are expected to present no significant fusion suppression as verified in the present work. This prediction does not take into account possible Coulomb breakup which may occur for very massive targets.

From the theoretical point of view, new approaches are available in the literature. We may mention the effect of continuum couplings on the fusion channel $(19,20)$.

Experiments are being carried out to measure the total reaction cross section (inelastic and transfer), as well as the breakup light particle channels for the ${ }^{6} \mathrm{Li}+{ }^{59} \mathrm{Co}$ and ${ }^{7} \mathrm{Li}+{ }^{59} \mathrm{Co}$ reactions. These measurements are fundamental to calibrate the coupling strength ot the breakup channel to the fusion channel, and must be auto consistent with the fusion suppression.

This work was supported in part by the Conselho Nacional de Desenvolvimento Científico e Tecnológico $(\mathrm{CNPq})$ and Fundação de Amparo à Pesquisa do Estado de São Paulo (FAPESP)-Brasil.

\section{References}

[1] S.G. Steadman and M.J. Rhoades-Brown, Annu. Rev. Nucl. Part. Sci. 36, 649 (1986).

[2] M. Beckerman Rep. Prog. Phys. 51, 1047 (1988).

[3] M. Dasgupta, D.J. Hinde, N. Rowley, A.M. Stefanini, Ann. Rev. Nucl. Part. Sci. 48, 401 and references there in

[4] C.H. Dasso,and A. Vitturi Phys. Rev. C50, R12 (1994) and C.H. Dasso, J.L. Guisado, S.M. Lenziand, A. Vitturi, Nucl. Phys. A597, 473 (1996).

[5] L.F. Canto, R. Donangelo, P. Lotti, and M.S. Hussein, Phys. Rev. C52, 1 (1995).

[6] N. Takigawa, M. Kuratani, H. Sagawa. Phys. Rev. C47, R2470 (1993).

[7] K. Hagino, Vitturi, C.H. Dasso, and S.M. Lenzi, Phys. Rev. C61, 037602 (2000).

[8] J. Takahashi, M. Munhoz, E.M. Szanto, N. Carlin, N. Added, A.A.P. Suaide, M.M. Moura, R. Liguori Neto, A. Szanto de Toledo, and L. F. Canto, Phys. Rev. Lett. 78, 30 (1997).

[9] C. Signorini, J. Phys. G Nucl. Part. Phys. 234, 1235 (1997) and Phys. Lett. B389, 457 (1996).

[10] K.E. Rehm, H. Esbensen, C.L. Jiang, B.B. Back, F. Borasi, B. Harss, R.V.F. Janssens, V. Nanal, J. Nolen, R.C. Pardo, M. Paul, P. Reiter, R. E. Segel, A. Sonzogni, J. Uusitalo, and A. H. Wuosmaa, Phys. Rev. Lett. 81, 3341 (1998).

[11] N. Dasgupta, D.J. Hinde, R.D. Butt, R.M. Anjos, A.C. Berriman, N. Carlin, P.R.S. Gomes, C.R. Morton, J.O. Newton, A. Szanto de Toledo, and K. Hagino, Phys. Rev. Lett 82, 1395 (1999).

[12] Vandana Tripathi, A. Navin, K. Mahata, K. Ramachandran, A. Chatterjee, and S. Kailas, Phys. Rev. Lett. 88, 172701-1 (2001).

[13] R. Cabezas, E.M. Szanto, N. Carlin, N. Added, A.A.P Suaide, M.M. de Moura, M. Munhoz, J. Takahashi, R. Liguori Neto, R.M. dos Anjos2, W.H.Z. Cardenas and A. Szanto de Toledo, Phys. Rev. C60, 067602 (1999).

[14] A.Szanto de Toledo, N. Added, W.H.Z. Cardenas, N. Carlin, M.M. de Moura, M.G. Munhoz, A.A.P. Suaide, E.M. Szanto, J. Takahashi, Nucl.Phys. A679, 175 (2000).

[15] R. Liguori Neto, J.C. Acquadro, P.R.S. Gomes, A. Szanto de Toledo, E. Crema, C. Tenreiro, N. Carlin, M.M. Carlin, and M.M. Coimbra, Nucl. Phys. A512, 333 (1990).

[16] W. Meczynski, P. Bednarczyk, R. Chapman, S. Courtin, J. Grebosz, F. Hannachi, P. Jones, J. Kownacki, M. Lach, A. Lopez-Martens, K.H. Maier, J.C. Merdinger, D. Middleton, M. Palacz, M.B. Smith, K.M. Spohr, N. Schulz, M. Zieblinski, and J.Styczen, Eur. Phys. J. A 3, 311 (1998).

[17] S. Courtin, Ph. D. Thesis, IReS Report (IReS 00-02) (2000).

[18] F. Pühlhofer, Nucl. Phys. A280, 267 (1977). 
[19] F.M. Nunes and I.J. Thompson, Phys. Rev. C 59, 2652 (1999).

02617 (2001).

[20] J. Tostevin, F.M. Nunes and I.J. Thompson, Phys. Rev. C 63 , 